Pacific Journal of Mathematics

TH ORDER INTEGRAL OPERATORS ASSOCIATED WITH 


\section{$n$th ORDER INTEGRAL OPERATORS ASSOCIATED WITH HILBERT TRANSFORMS}

\section{G. O. OKIKIOLU}

In this paper, we study the $n$th order analogues of certain integral operators allied to the Hilbert transform and to Dirichlet's integrals. Most of the results known to be true for $n=0$ are proved for the general case. Some cases in which the analogy fails are also considered. Among the integrals considered are transforms $B_{\alpha}^{(n)}(f)$ and $I_{\alpha}^{(n)}(f)$ defined by

$$
\begin{aligned}
B_{\alpha}^{(n)}(f)=(-1)^{n} & \frac{(2 n+1) !}{\pi}(\mathrm{P} . \mathrm{V} .) \int_{-\infty}^{\infty} f(t) \\
& \times \frac{\sin \alpha(t-x)-\sum_{m=0}^{n-1} \frac{(-1)^{m}(\alpha t-\alpha x)^{2 m+1}}{(2 m+1) !}}{(t-x)^{2 n+2}} d t \\
I_{\alpha}^{(n)}(f)=(-1)^{n} & \frac{(2 n+2) !}{\pi}(\mathrm{P} . \mathrm{V} .) \int_{-\infty}^{\infty} f(t) \\
& \times \frac{\sum_{m=0}^{n} \frac{(-1)^{m}(\alpha t-\alpha x)^{2 m}}{(2 m) !}-\cos \alpha(t-x)}{(t-x)^{2 n+3}} d t .
\end{aligned}
$$

Inversion processes by which $f$ may be expressed in terms of the $B_{a}^{(n)}$ and $I_{\alpha}^{(n)}$ operators are also obtained. The results proved in the paper are also shown to be true for integrals defined with respect to a continuous parameter $\nu$. These integrals reduce to the original ones when $\nu$ is an integer.

Let the functions $c_{n}^{(1)}(x), s_{n}^{(1)}(x), c_{n}(x)$ and $s_{n}(x)$ be defined as follows:

$$
\begin{aligned}
c_{n}^{(1)}(x) & =\sum_{m=0}^{n} \frac{(-1)^{m} x^{2 m}}{(2 m) !}, n \geqq 0, c_{-1}^{(1)}(x)=0, \\
s_{n}^{(1)}(x) & =\sum_{m=0}^{n-1} \frac{(-1)^{m} x^{2 m+1}}{(2 m+1) !}, n \geqq 1, s_{0}^{(1)}(x)=0, \\
s_{n}(t) & =\frac{(2 n) !}{\pi}(-1)^{n} \frac{\sin \alpha t-s_{n}^{(1)}(\alpha t)}{t^{2 n+1}} \\
c_{n}(t) & =\frac{(2 n) !}{\pi}(-1)^{n} \frac{c_{n}^{(1)}(\alpha t)-\cos \alpha t}{t^{2 n+1}},
\end{aligned}
$$

where $\alpha$ is a fixed positive number. Then we define the following integrals. 


$$
\begin{aligned}
& D_{a}^{(n)}(f)(x)=\int_{\infty}^{\infty} f(t) s_{n}(t-x) d t, \\
& S_{a}^{(n)}(f)(x)=\int_{\infty}^{\infty} f(t) c_{n}(t-x) d t, \\
& C_{a}^{(n)}(f)(x)=(2 n+1) \int_{-\infty}^{\infty} f(t) \frac{c_{n}(t-x)}{t-x} d t, \\
& B_{a}^{(n)}(f)(x)=(2 n+1)(\mathrm{P} . \mathrm{V} \cdot) \int_{-\infty}^{\infty} f(t) \frac{s_{n}(t-x)}{(t-x)} d t, \\
& I_{\infty}^{(n)}(f)(x)=(2 n+2)(2 n+1)(\mathrm{P} . \mathrm{V} \cdot) \int_{-\infty}^{\infty} f(t) \frac{c_{n}(t-x)}{(t-x)^{2}} d t .
\end{aligned}
$$

$D_{\alpha}^{(n)}, S_{\alpha}^{(n)}, C_{\alpha}^{(n)}$ and $B_{\alpha}^{(n)}$ are defined for integers $n \geqq 0$, and $I_{\alpha}^{(n)}$ for $n \geqq-1$. The operators $D_{\alpha}^{(0)}, S_{\alpha}^{(0)}, C_{\alpha}^{(0)}, B_{\alpha}^{(0)}$ and $I_{\alpha}^{(0)}$ will be denoted by $D_{\alpha}, S_{\alpha}, C_{\alpha}, B_{\alpha}$ and $I_{\alpha}$ respectively.

The above integrals are generalisations of known integral operators. $D_{\alpha}(f)$ being the Dirichlet integral, and $S_{\alpha}(f)$ its well-known 'conjugate'. $B_{\alpha}(f)$ was introduced by Boas in [1], and has since been studied by Goldberg [2], Heywood [3] and Kober [4]. The transform $I_{\alpha}(f)$ has been studied by the author elsewhere. The integrals are all related to Hilbert and Fourier transforms, and the properties of $B_{\alpha}(f)$ and $I_{\alpha}(f)$ have been obtained from identities involving the transforms and the operators $D_{\alpha}$ and $S_{\alpha}$.

In this paper we obtain results involving $D_{\alpha}^{(n)}(f)$ and $S_{\alpha}^{(n)}(f)$ similar to those of $D_{\alpha}(f)$ and $S_{\alpha}(f)$, and use these in studying the transforms $B_{\alpha}^{(n)}(f)$ and $I_{\alpha}^{(n)}(f)$. Among the results obtained are inversion processes by which $f$ may be expressed in terms of $B_{a}^{(n)}(f)$ and $I_{a}^{(n)}(f)$. In Section 7 , we show that the exponent $n$ may be replaced by a continuous real (or complex) variable. This is done by replacing the kernels defining the integrals by certain others which reduce to the ones already given when the exponent is an integer. The integrals $D_{\alpha}^{(\nu)}$ so obtained define a semi-group of operators.

We shall consider functions of the class $L^{p}(-\infty, \infty)(p>1)$ only. This class will in general be denoted by $L^{p}$. The positive numbers $p$ and $p^{\prime}$ will be connected by the equation $1 / p+1 / p^{\prime}=1$, and the expression

$$
\left(\int_{-\infty}^{\infty}|f(t)|^{p} d t\right)^{1 / p}
$$

will be denoted by $\|f\|_{p}$. Most of the equations and identities of this paper should be taken as true almost everywhere. This will not be indicated in each case. The Hilbert transform is defined by

$$
H(f)(x)=\frac{1}{\pi}(\mathrm{P} . \mathrm{V} .) \int_{-\infty}^{\infty} \frac{f(t)}{t-x} d t,
$$


and the Fourier transform $\mathscr{F}(f)$ of a function $f \in L^{p}(1 \leqq p \leqq 2)$ is given by

$$
\hat{f}(x)=\mathscr{F}(f)(x)=\frac{1}{\sqrt{2 \pi}} \underset{\text { lorder } \left.p^{\prime}\right)}{\text { l.i.m }} \int f(t) e^{i x t} d t .
$$

2. The properties of $D_{\alpha}^{(n)}(f)$ and $S_{a}^{(n)}(f)$. The following lemmas will be employed in the proofs of the results of this section.

Lemma 1. (a) $H\left(s_{n}\right)(x)=-c_{n}(x)$, (b) $H\left(c_{n}\right)(x)=s_{n}(x)$.

Proof. Consider the function

$$
g(z)=\frac{\sum_{m=0}^{2 n} \frac{(i z)^{m}}{m !}-e^{i \alpha z}}{z^{2 n+1}}, \quad z=x+i y,
$$

where $g=g(x), x$ real. $g(z)$ is a regular function for $y \geqq 0$, and tends to 0 uniformly for $0 \leqq \arg z \leqq \pi$ as $|z| \rightarrow \infty$. Hence by integrating $g(z) / z-\xi$ round the semi-circle of radius $R$ in the upper half plane indented at $t=\xi$, we have

$$
\left(\int_{\xi+\delta}^{R}+\int_{-R}^{\xi-\delta}\right) \frac{g(t)}{t-\xi} d t+i \int_{0}^{\pi} \frac{g\left(R e^{i \phi}\right)}{R e^{i \phi}-\xi} R \in{ }^{i \phi} d \phi-i \int_{0}^{\pi} g\left(\xi+\delta e^{i \phi}\right) d \phi=0 .
$$

On letting $\delta \rightarrow 0$ and $R \rightarrow \infty$, we obtain

$$
H(g)(\xi)=i g(\xi) \text {. }
$$

The results (a) and (b) follow by equating real and imaginary parts of this identity.

LEMMA 2.

(a) $\sqrt{2 \pi} s_{n}(x-y)=\frac{1}{\sqrt{2 \pi}} \int_{\alpha-}^{\alpha}(\alpha-|t|)^{2 n} e^{i(x-y) t} d t$,

(b) $i \sqrt{2 \pi} c_{n}(x-y)=\frac{1}{\sqrt{2 \pi}} \int_{-\alpha}^{\alpha}(\alpha-|t|)^{2 n} \frac{|t|}{t} e^{i(x-y) t} d t$.

Proof. Let

$$
I_{n}=\frac{i^{n}}{n !} \int_{0}^{\alpha}(\alpha-t)^{n} e^{i x t} d t
$$

Then it is easy to verify by integrating by parts that

$$
x^{n} I_{n}-x^{n-1} I_{n-1}=-\frac{(i x \alpha)^{n}}{i x n !} .
$$


Hence by making appropriate summation and putting $2 n$ for $n$, we obtain

$$
\frac{(-1)^{n}}{(2 n) !} \int_{0}^{\alpha}(\alpha-t)^{2 n} e^{i x t} d t=-\frac{\sum_{m=0}^{2 n} \frac{(i x \alpha)^{m}}{m !}-e^{i x \alpha}}{i x^{2 n+1}} .
$$

A similar expression is obtained by putting $-\alpha$ for $\alpha$, and the results of the lemma are then easily verified.

LEMMA 3. Let

$$
h(x)=\int_{-\infty}^{\infty} f(t) g(x-t) d t .
$$

If $f \in L^{p}(p \geqq 1), g \in L^{q}(q \geqq 1)$, where $1 / p+1 / q \geqq 1$, then

$$
\|h\|_{r} \leqq\|f\|_{p}\|g\|_{q},
$$

where $1 / r=1 / p+1 / q-1$.

Proof. This is a well-known result, a proof of which is given in Lemma $\beta$, p. 97, of [5]. (The case in which $p=1$ or $q=1$ is obtained similarly).

In the next theorem, we give expressions for $D_{\alpha}^{(n)}(f)$ and $S_{\infty}^{(n)}(f)$ in terms of $\hat{f}$. These results will be applied later in calculating the integrals for certain special cases.

THEOREM 1. If $f \in L^{p}$ where $1<p \leqq 2$, then for $n \geqq 0$,

(a) $D_{\alpha}^{(n)}(f)(x)=\frac{1}{\sqrt{2 \pi}} \int_{-\alpha}^{\alpha}(\alpha-|t|)^{2 n} \hat{f}(t) e^{-i x t} d t$,

(b) $S_{\alpha}^{(n)}(f)(x)=\frac{-1}{\sqrt{2 \pi}} \int_{-\alpha}^{\alpha} \frac{|t|}{t}(\alpha-t)^{2 n} \hat{f}(t) e^{-i x t} d t$.

Proof. Let

$$
g_{2}(x)=(\alpha-|x|)^{2 n} e^{-i y x} \quad \text { for } \quad x \in(-\alpha, \alpha) ;=0 \text { otherwise , }
$$

and let

$$
g_{1}(x)=\frac{|x|}{x}(\alpha-|x|)^{2 n} e^{-i y x} \quad \text { for } \quad x \in(-\alpha, \alpha) ;=0 \text { otherwise . }
$$

Then $\widehat{g}_{1}$ and $\hat{g}_{2}$ are given in Lemma 2. Hence Theorem 1 follows by applying the product formula for Fourier transforms (Theorems 49 and 75 of [5]).

CoROLLARY 1.1. If $f \in L^{p}$ where $1<p \leqq 2$, then for $n \geqq 0$, 


$$
D_{\alpha}^{(n)}(f)=0 \text { if and only if } \hat{f}(x)=0 \text { for } x \in(-\alpha, \alpha)
$$

and

$$
S_{\alpha}^{(n)}(f)=0 \text { if and only if } \hat{f}(x)=0 \text { for } x \in(-\alpha, \alpha) .
$$

Proof. This obviously follows from Theorem 1.

Corollary 1.2. Let $m \geqq 0$ and $n \geqq 0$. Then

(a) $\quad D_{a}^{(m)}\left(s_{n}\right)(x)=S_{a}^{(m)}\left(c_{n}\right)(x)=s_{n+m}(x)$,

(b) $\quad-S_{a}^{(m)}\left(s_{n}\right)(x)=D_{a}^{(m)}\left(c_{n}\right)(x)=c_{n+m}(x)$.

Proof. Let $g_{1}$ and $g_{2}$ be defined as in the proof of Theorem 1 with $y=0$. Then by Lemma 2 , we have

$$
\widehat{g}_{1}(x)=\sqrt{2 \pi} s_{n}(x) \text { and } \hat{g}_{2}(x)=i \sqrt{2 \pi} c_{n}(x) .
$$

Hence by the inversion for Fourier transforms it follows that

$$
\sqrt{2 \pi} \hat{s}_{n}(x)=(\alpha-|x|)^{2 n} \text { for } x \in(-\alpha, \alpha) ;=0 \text { otherwise }
$$

and

$$
-i \sqrt{2 \pi} \hat{c}_{n}(x)=\frac{|x|}{x}(\alpha-|x|)^{2 n} \text { for } x \in(-\alpha, \alpha) ;=0 \text { otherwise . }
$$

By putting $s_{n}$ for $f$ and writing $m$ for $n$ in Theorem 1, we have

$$
\begin{aligned}
& D_{\alpha}^{(m)}\left(s_{n}\right)(x)=\frac{1}{2 \pi} \int_{-\alpha}^{\alpha}(\alpha-|t|)^{2 m+2 n} e^{i x t} d t=s_{m+n}(x), \\
& S_{\alpha}^{(m)}\left(s_{n}\right)(x)=\frac{+i}{2 \pi} \int_{-\alpha}^{\alpha} \frac{|t|}{t}(\alpha-|t|)^{2 m+2 n} e^{i x t} d t=-c_{m+n}(x) .
\end{aligned}
$$

The results involving $c_{n}$ follow by proceeding similarly.

THEOREM 2. If $f \in L^{p}$ where $p>1$, then for $n \geqq 0$, we have

$$
D_{a}^{(n)}(f) \in L^{r} \quad \text { and } \quad S_{a}^{(n)}(f) \in L^{r} \quad \text { for } r \geqq p .
$$

Also, for $g \in L^{r^{\prime}}$, we have

$$
\begin{aligned}
& \int_{-\infty}^{\infty} g(t) D_{\alpha}^{(n)}(f)(t) d t=\int_{-\infty}^{\infty} f(t) D_{\alpha}^{(n)}(g)(t) d t, \\
& \int_{-\infty}^{\infty} g(t) S_{\alpha}^{(n)}(f)(t) d t=-\int_{-\infty}^{\infty} f(t) S_{\alpha}^{(n)}(g)(t) d t .
\end{aligned}
$$

Proof. Since $s_{n}(x)=0(1)$ as $x \rightarrow 0$ and is $0\left(1 / x^{2}\right)$ as $x \rightarrow \infty$ if $n \geqq 1$, it belongs to $L^{q}$ for $q \geqq 1$. Hence by Lemma 3 , we have 


$$
D_{\alpha}^{(n)}(f) \in L^{r} \quad \text { for } r \geqq p \quad \text { if } \quad n \geqq 1 \text {. }
$$

For $n=0$,

$$
\begin{aligned}
D_{\alpha}(f)(x)= & \frac{\cos \alpha x}{\pi}(\mathrm{P} . \mathrm{V} .) \int_{-\infty}^{\infty} \frac{f(t) \sin \alpha t}{t-x} d t \\
& -\frac{\sin \alpha x}{\pi}(\mathrm{P} . \mathrm{V} .) \int_{-\infty}^{\infty} \frac{f(t) \cos \alpha t}{t-x} d t
\end{aligned}
$$

It is thus clear from Theorem 101 of [5] that $D_{\alpha}(f) \in L^{p}$. The rerult $D_{\alpha}(f) \in L^{r}$ for $r>p$ follows from Lemma 3 since $s_{0}(x) \in L^{q}$ for $q>1$. It is easy to verify that $c_{n}(x) \in L^{q}$ for $q>1$. Hence we also have

$$
S_{\alpha}^{(n)}(f) \in L^{r} \text { for } r>p
$$

In Theorem 4 (below) we prove that $S_{a}^{(n)}(f)=H\left\{D_{a}^{(n)}(f)\right\}$. Hence it follows from Theorem 101 of [5] that $S_{a}^{(n)}(f) \in L^{p}$. Now suppose that $g \in L^{r^{\prime}}$. Then we have

$$
\begin{aligned}
\int_{-\infty}^{\infty} g(x) D_{\infty}^{(n)}(f)(x) d x & =\int_{-\infty}^{\infty} g(x) d x \int_{-\infty}^{\infty} f(t) s_{n}(t-x) d t \\
& =\int_{-\infty}^{\infty} f(t) d t \int_{-\infty}^{\infty} g(x) s_{n}(x-t) d x=\int_{-\infty}^{\infty} f(t) D_{\infty}^{(n)}(g)(t) d t,
\end{aligned}
$$

the inversion of the integrals being justified by absolute convergence. The proof of the second product formula follows similarly.

THEOREM 3. If $f \in L^{p}(p>1)$, then for $m \geqq 0, n \geqq 0$, we have

(a) $D_{\alpha}^{(m)}\left\{D_{\alpha}^{(n)}(f)\right\}(x)=-S_{\alpha}^{(m)}\left\{S_{\alpha}^{(n)}(f)\right\}(x)=D_{\alpha}^{(m+n)}(f)(x)$,

(b) $S_{\alpha}^{(m)}\left\{D_{\alpha}^{(n)}(f)\right\}(x)=D_{\alpha}^{(m)}\left\{S_{\alpha}^{(n)}(f)\right\}(x)=S_{\alpha}^{(m+n)}(f)(x)$.

Proof. By putting $g(x)=s_{n}(x-y)$ in the product formulae (1) and (2) and using Corollary 1.2, we have

$$
\begin{aligned}
& \int_{-\infty}^{\infty} s_{n}(x-y) D_{\alpha}^{(m)}(f)(x) d x=\int_{-\infty}^{\infty} f(t) s_{m+n}(t-y) d t, \\
& \int_{-\infty}^{\infty} s_{n}(x-y) S_{\alpha}^{(m)}(f)(x) d x=\int_{-\infty}^{\infty} f(t) c_{m+n}(t-y) d t ;
\end{aligned}
$$

i.e.

$$
D_{\alpha}^{(n)}\left\{D_{\alpha}^{(m)}(f)\right\}(y)=D_{\alpha}^{(m+n)}(f)(y), D_{\alpha}^{(n)}\left\{S_{\alpha}^{(m)}(f)\right\}(y)=S_{\alpha}^{(m+n)}(f)(y) .
$$

The other results of the theorem can be obtained by considering $c_{n}(x)$ in place of $s_{n}(x)$.

COROLLARY 3.1. Let $f \in L^{p}(p>1)$ and let $l \geqq n$ be integers. (a) If $D_{\alpha}^{(n)}(f)=0$, then $D_{\alpha}^{(l)}(f)=S_{\alpha}^{(l)}(f)=0$, 
(b) If $S_{a}^{(n)}(f)=0$, then $S_{a}^{(l)}(f)=D_{a}^{(l)}(f)=0$.

Proof. These results are obvious consequences of Theorem 3.

REMARK 1. We observe here that Corollary 1.1 implies that if $f \in L^{p}$ where $1<p \leqq 2$, then $D_{a}^{(n)}(f)=0$ implies $D_{a}^{(l)}(f)=S_{\alpha}^{(l)}(f)=0$ for all $1 \geqq 0$, and so also for $S_{a}^{(n)}(f)$. In Section 6 , we show that this is the case for $f \in L^{p}(p>1)$.

THEOREM 4. If $f \in L^{p}(p>1)$, then for $n \geqq 0$,

(a) $D_{\alpha}^{(n)}\{H(f)\}=H\left\{D_{\alpha}^{(n)}(f)\right\}=S_{\alpha}^{(n)}(f)$,

(b) $S_{a}^{(n)}\{H(f)\}=H\left\{S_{a}^{(n)}(f)\right\}=-D_{a}^{(n)}(f)$.

Proof. From the product formula for Hilbert transforms (Theorem 102 of [5]) and Lemma 1, we clearly have

$$
\int_{-\infty}^{\infty} s_{n}(t-y) f(t) d t=-\int_{-\infty}^{\infty} H(f)(t) c_{n}(t-y) d t
$$

and

$$
\int_{-\infty}^{\infty} c_{n}(t-y) f(t) d t=\int_{-\infty}^{\infty} H(f)(t) s_{n}(t-y) d t
$$

We shall now show that $D_{\alpha}^{(n)}\{H(f)\}=H\left\{D_{\alpha}^{(n)}(f)\right\}$. The corresponding result for $S_{\alpha}^{(n)}$ follows similarly. Let $\delta>0$ and let $b<\infty$. Then by the absolute convergence of the integrals involved, we have

$$
\int_{x+\delta}^{\infty} \frac{d x}{x-y} \int_{-b}^{b} f(t+x-y) s_{n}(t-y) d t=\int_{-b}^{b} s_{n}(t-y) d t \int_{t+\delta}^{\infty} \frac{f(x)}{x-t} d x
$$

and

$$
\int_{-\infty}^{x-\delta} \frac{d x}{x-y} \int_{-b}^{b} f(t+x-y) s_{n}(t-y) d t=\int_{-b}^{b} s_{n}(t-y) d t \int_{-\infty}^{t-\delta} \frac{f(x)}{x-t} d t .
$$

Let $\left(\int_{\xi+\delta}^{\infty}+\int_{-\infty}^{\xi-\delta}\right)$ be denoted by $\int_{-\infty}^{1 \infty}$. Then on letting $b \rightarrow \infty$, we obtain

$$
\int_{-\infty}^{1 \infty} \frac{d x}{x-y} D_{\alpha}^{(n)}(f)(x) d x=\int_{-\infty}^{\infty} s_{n}(t-y) d t \int_{-\infty}^{1 \infty} \frac{f(x)}{x-t} d x
$$

Since $s_{n}(t) \in L^{p}$ for $q>1$, and since

$$
\lim _{\delta \rightarrow 0}\left\|H(f)(t)-\frac{1}{\pi} \int_{-\infty}^{\infty} \frac{f(x)}{x-t} d x\right\|_{p}=0,
$$

the required result follows by letting $\delta \rightarrow 0$. 
REMARK 2. It is obvious from Theorem 4 that $D_{\alpha}^{(n)}(f)=0$ if and only if $D_{\alpha}^{(n)}\{H(f)\}=0$, with a similar result for $S_{\alpha}^{(n)}(f)$.

We shall now consider the equation $D_{\alpha}^{(n)}(f)=\lambda f$. For the case $n=0$, we have $D_{\alpha}(f)=D_{\alpha}\left\{D_{\alpha}(f)\right\}=\lambda D_{\alpha}(f)$, so that $\lambda=1$. It has been shown (for example see Lemma 3(b) of [4]) that in this case, $f$ is a member of the class $G_{\alpha}^{p}$ of functions $f(x) \in L^{p}$ such that $f(z)$ $(z=x+i y)$ is an entire function of exponential order $\geqq \alpha$. For $n>0$ however, we show in the next theorem that there is no nontrivial member of $L^{p}(1<p \leqq 2)$ satisfying the equation. Further, we show that in any case, we must have $|\lambda|<1$.

THEOREM 5. Suppose that $f$ satisfies the equation

$$
D_{\alpha}^{(n)}(f)=\lambda f \text { where } n>0 \text { and } \lambda \neq 0 \text {. }
$$

(a) If $f \in L^{p}(1<p \leqq 2)$, then $f=0$.

(b) If $f \in L^{p}(p>2)$, then $|\lambda|<1$.

Proof. Suppose that $f \in L^{p}(1<p \leqq 2)$. Then by applying Theorem 1(a), we have

$$
\frac{1}{\sqrt{2 \pi}} \int_{-\alpha}^{\alpha}(\alpha-|t|)^{2 n} \hat{f}(t) e^{-i x t} d t=\lambda f(x)
$$

so that

$$
\lambda \hat{f}(x)=\left\{\begin{array}{cc}
(\alpha-|x|)^{2 n} \hat{f}(x) & \text { for }|x|<\alpha \\
0 & \text { for }|x|>\alpha
\end{array} .\right.
$$

It is now obvious that $\hat{f}=0$, and hence that $f=0$ by the uniqueness of Fourier transforms.

Now consider the general case in which $f \in L^{p}(p>1)$. Then we clearly have

$$
D_{\alpha}^{(2 n)}(f)=D_{\alpha}^{(n)}\left\{D_{\alpha}^{(n)}(f)\right\}=\lambda D_{\alpha}^{(n)}(f)=\lambda^{2} f,
$$

and it follows by induction that for every positive integer $k$,

$$
D_{\alpha}^{(k n)}(f)=\lambda^{k} f \text {. }
$$

Now from Lemma 3, it is clear that

$$
\left\|D_{\alpha}^{(k n)}(f)\right\|_{r} \leqq\|f\|_{p}\left\|s_{k n}\right\|_{q}
$$

where $q \geqq 1,1 / p+1 / q \geqq 1$ and $1 / r=1 / p+1 / q-1$. It is however not difficult to see that for $q \geqq 1$

$$
\lim _{k \rightarrow \infty}\left\|s_{k n}\right\|_{q}=0
$$


so that

$$
\lim _{k \rightarrow \infty}\left\|D_{a}^{(k n)}(f)\right\|_{r}=0 \quad \text { for } r \geqq p .
$$

Since $D_{\alpha}^{(k n)}(f)=\lambda^{k} f$ however, we must have $|\lambda|<1$ or $\|f\|_{p}=0$. The result (b) follows.

COROLlary 5.1. Let $f \in L^{p}(p<1)$ and let $m>0$ and $n \geqq 0$ be integers. Then $D_{a}^{(m+n)}(f)=D_{\alpha}^{(n)}(f)$ if and only if $D_{a}^{(n)}(f)=0$.

Proof. By Theorem 3(a), we have

$$
D_{\alpha}^{(m)}\left\{D_{\alpha}^{(n)}(f)\right\}=D_{\alpha}^{(m+n)}(f)=D_{\alpha}^{(n)}(f) .
$$

The result clearly follows from Theorem 5 .

3. Representation theorems for $B_{a}^{(n)}(f)$ and $I_{a}^{(n)}(f)$. We shall now obtain results by which $B_{\alpha}^{(n)}(f)$ and $I_{\alpha}^{(n)}(f)$ are expressed in terms of $D_{\alpha}^{(n)}(f)$ and $S_{\alpha}^{(n)}(f)$.

LEMmA 4. Let $f \in L^{p}(p>1)$ and let $g \in L^{p^{\prime}}$. If $\int_{-\infty}^{\infty} f(t) \frac{d}{d x} g(t-x) d t$ belongs to $L^{r}$ for some number $r>1$, then

$$
\frac{d}{d x} \int_{-\infty}^{\infty} f(t) g(t-x) d t=\int_{-\infty}^{\infty} f(t) \frac{d}{d x} g(t-x) d t
$$

Proof. Let $x$ be a fixed finite number. Then by absolute convergence, we have

$$
\begin{aligned}
\int_{0}^{x} d y \int_{-\infty}^{\infty} f(t) \frac{d}{d y} g(t-y) d t & =\int_{-\infty}^{\infty} f(t) d t \int_{0}^{x} \frac{d}{d y} g(t-y) d y \\
& =\int_{-\infty}^{\infty} f(t)\{g(t-x)-g(t)\} d t
\end{aligned}
$$

and the required result follows immediately.

The following identities can be obtained directly from the definition.

$$
\begin{aligned}
I_{\alpha}^{(n)}(f) & =\alpha^{2 n+2} H(f)-S_{\alpha}^{(n+1)}(f) \\
I_{\alpha}^{(-1)}(f) & =H(f)-S_{\alpha}(f) \\
\frac{d}{d x} c_{n}^{(1)}(x) & =-s_{n}^{(1)}(x) ; \frac{d}{d x} s_{n}^{(1)}(x)=c_{n-1}^{(1)}(x)
\end{aligned}
$$

LEMMA 5. If $f \in L^{p}(p>1)$, then $C_{\alpha}^{(n)}(f) \in L^{r}$ for $r \geqq p$, and 


$$
C_{\alpha}^{(n)}(f)=\alpha D_{\alpha}^{(n)}(f)+\frac{d}{d x} S_{\alpha}^{(n)}(f)
$$

Proof. We have the identity

$$
\frac{d}{d x} c_{n}(t-x)=-\alpha s_{n}(t-x)+(2 n+1) \frac{c_{n}(t-x)}{t-x} .
$$

Since $-\alpha s_{n}(t)+(2 n+1) c_{n}(t) / t$ belongs to $L^{q}$ for $q \geqq 1$, it is clear from Lemma 3 that

$$
\int_{-\infty}^{\infty} f(t) \frac{d}{d x} c_{n}(t-x) d t
$$

belongs to $L^{r}$ for $r \geqq p$. Hence it follows that $C_{\alpha}^{(n)}(f) \in L^{r}$ for $r \geqq p$. The identity of the lemma then follows from Lemma 4 .

THEOREM 6. If $f \in L^{p}(p<1)$, then $B_{\infty}^{(n)}(f) \in L^{p}$, and

$$
B_{\alpha}^{(n)}(f)=\alpha^{2 n+1} H(f)-\alpha S_{\alpha}^{(n)}(f)+\frac{d}{d x} D_{\alpha}^{(n)}(f) .
$$

Proof. Since

$$
(-1)^{n} \alpha(2 n) ! \frac{c_{n}^{(1)}(\alpha x)-\cos \alpha x-(-1)^{n} \frac{(\alpha x)^{2 n}}{(2 n) !}}{x^{2 n+1}}+(2 n+1) \frac{s_{n}(x)}{x}
$$

belongs to $L^{q}$ for $q \geqq 1$, it follows from Lemma 3 and from obvious identities that

$$
\alpha S_{\alpha}^{(n)}(f)-\alpha^{2 n+1} H(f)+B_{\alpha}^{(n)}(f)=\int_{-\infty}^{\infty} f(t) \frac{d}{d x} s_{n}(t-x) d t,
$$

and that this belongs to $L^{r}$ for $r \geqq p$. Hence by Lemma 4,

$$
\alpha S_{\alpha}^{(n)}(f)-\alpha^{2 n+1} H(f)+B_{\alpha}^{(n)}(f)=\frac{d}{d x} D_{\alpha}^{(n)}(f) \in L^{p} .
$$

The theorem now follows from Theorem 2 and from the fact that $H(f) \in L^{p}$.

Theorem 7. If $f \in L^{p}(p>1)$, then $I_{\alpha}^{(n)}(f) \in L^{p}$, and

$$
I_{\alpha}^{(n)}(f)=\alpha^{2 n+2} H(f)-\alpha^{2} S_{\alpha}^{(n)}(f)+2 \alpha \frac{d}{d x} D_{a}^{(n)}(f)+\frac{d^{2}}{d x^{2}} S_{a}^{(n)}(f) .
$$

Proof. By using the identity 


$$
\begin{aligned}
(2 n+1) \frac{d}{d x} \frac{c_{n}(t-x)}{t-x}= & -\alpha(2 n+1) \frac{s_{n}(t-x)}{t-x} \\
& +(2 n+1)(2 n+2) \frac{c_{n}(t-x)}{(t-x)^{2}}
\end{aligned}
$$

and proceeding as in previous cases (Lemma 5 and Theorem 6), we have

$$
-\alpha B_{\alpha}^{(n)}(f)+I_{\alpha}^{(n)}(f)=\frac{d}{d x} C_{\alpha}^{(n)}(f) \in L^{r}
$$

for $r \geqq p$, so that $I_{\alpha}^{(n)}(f) \in L^{p}$ (by Theorem 6).

Next by substituting for $C_{\alpha}^{(n)}(f)$ and $B_{\alpha}^{(n)}(f)$ from Lemma 5 and Theorem 6 respectively, we derive the identity given in the theorem.

CoROLLARY 6.1. If $f \in L^{p}(p>1)$, then

$$
S_{\alpha}^{(n+1)}(f)=\alpha^{2} S_{\alpha}^{(n)}(f)-2 \alpha \frac{d}{d x} D_{\alpha}^{(n)}(f)-\frac{d^{2}}{d x^{2}} S_{\alpha}^{(n)}(f) .
$$

Proof. This is easily verified by substituting for $I_{\alpha}^{(n)}(f)-\alpha^{2 n+2} H(f)$ in (3).

Note. An expression similar to that given in Corollary 6.1 can be obtained for $D_{a}^{(n+1)}(f)$ by putting $H(f)$ for $f$ and using Theorem 4.

By substituting $D_{\alpha}^{(m)}(f), S_{\alpha}^{(m)}(f)$ and $H(f)$ respectively for $f$ in the identities of Theorems 6 and 7, and using Theorems 3 and 4, we have the following:

$$
\begin{aligned}
B_{\alpha}^{(n)}\left\{D^{(m)}(f)\right\}= & \alpha^{2 n+1} S_{\alpha}^{(m)}(f)-\alpha S_{\alpha}^{(m+n)}(f)+\frac{d}{d x} D_{\alpha}^{(m+n)}(f) \\
B_{\alpha}^{(n)}\left\{S_{\alpha}^{(m)}(f)\right\}= & -\alpha^{2 n+1} D_{\alpha}^{(m)}(f)+\alpha D_{\alpha}^{(m+n)}(f)+\frac{d}{d x} S_{\alpha}^{(m+n)}(f) \\
B_{\alpha}^{(n)}\{H(f)\}= & -\alpha^{2 n+1} f+\alpha D_{\alpha}^{(n)}(f)+\frac{d}{d x} S_{\alpha}^{(n)}(f) \\
I_{\alpha}^{(n)}\left\{D_{\alpha}^{(m)}(f)\right\}= & \alpha^{2 n+2} S_{\alpha}^{(m)}(f)-\alpha^{2} S_{\alpha}^{(m+n)}(f) \\
& +2 \alpha \frac{d}{d x} D_{\alpha}^{(m+n)}(f)+\frac{d^{2}}{d x^{2}} S_{\alpha}^{(m+n)}(f) \\
I_{\alpha}^{(n)}\left\{S_{\alpha}^{(m)}(f)\right\}= & -\alpha^{2 n+2} D_{\alpha}^{(m)}(f)+\alpha^{2} D_{\alpha}^{(m+n)}(f) \\
& +2 \alpha \frac{d}{d x} S_{\alpha}^{(m+n)}(f)-\frac{d^{2}}{d x^{2}} D_{\alpha}^{(m+n)}(f) \\
I_{\alpha}^{(n)}\{H(f)\}= & -\alpha^{2 n+2} f+\alpha^{2} D_{\alpha}^{(n)}(f) \\
& +2 \alpha \frac{d}{d x} S_{\alpha}^{(n)}(f)-\frac{d^{2}}{d x^{2}} D_{\alpha}^{(n)}(f)
\end{aligned}
$$


The results of the next theorem will be applied in deriving the inversion processes for $B_{\alpha}^{(n)}(f)$ and $I_{\alpha}^{(n)}(f)$.

THEOREM 8. If $f \in L^{p}(p>1)$ and if $f$ satisfies $D_{\alpha}^{(m+n)}(f)=D_{a}^{(n)}(f)$ for some number $m \geqq 0$, then

$$
\begin{aligned}
B_{\alpha}^{(n)}\left\{D_{\alpha}^{(m)}(f)-f\right\} & =\alpha^{2 n+1}\left\{S_{\alpha}^{(m)}(f)-H(f)\right\}, \\
B_{\alpha}^{(n)}\left\{S_{\alpha}^{(m)}(f)-H(f)\right\} & =-\alpha^{2 n+1}\left\{D_{\alpha}^{(m)}(f)-f\right\} . \\
I_{\alpha}^{(n)}\left\{D_{\alpha}^{(m)}(f)-f\right\} & =\alpha^{2 n+2}\left\{S_{\alpha}^{(m)}(f)-H(f)\right\}, \\
I_{\alpha}^{(n)}\left\{S_{\alpha}^{(m)}(f)-H(f)\right\} & =-\alpha^{2 n+2}\left\{D_{\alpha}^{(m)}(f)-f\right\} .
\end{aligned}
$$

Proof. It is quite easy to verify these results from the identities (6) -(11) by applying Corollary 5.1. We observe that the condition $D_{\alpha}^{(m+n)}(f)=D_{\alpha}^{(n)}(f)$ is an identity if $m=0$, and for $m>0$, it implies that

$$
D_{a}^{(m+n)}(f)=D_{a}^{(n)}(f)=0 .
$$

4. Product formulae and commutative property. It is clear that $B_{\alpha}^{(n)}(f)-\alpha^{2 n+1} H(f)$ and $I_{\alpha}^{(n)}(f)-\alpha^{2 n+2} H(f)$ are absolutely and uniformly convergent integrals. Hence for $g \in L^{p^{\prime}}$, we have

$$
\begin{aligned}
\int_{-\infty}^{\infty} g(t)\left\{B_{\alpha}^{(n)}(f)(t)-\alpha^{2 n+1} H(f)(t)\right\} d t \\
\quad=\int_{-\infty}^{\infty} g(t) d t \int_{-\infty}^{\infty} f(x) \frac{s_{n}(x-t)-\alpha^{2 n+1}(x-t)}{(x-t)} d x \\
=\int_{-\infty}^{\infty} f(x) d x \int_{-\infty}^{\infty} g(t) \frac{s_{n}(t-x)-\alpha^{2 n+1}(t-x)}{(t-x)} d t \\
=-\int_{-\infty}^{\infty} f(x)\left\{B_{\alpha}^{(n)}(g)(x)-\alpha^{2 n+1} H(g)(x)\right\} d x .
\end{aligned}
$$

A similar result holds for $I_{\alpha}^{(n)}(f)-\alpha^{2 n+2} H(f)$.

Hence by the product formula for Hilbert transforms (Theorem 102 of [5]), we have

\section{THEOREM 9. If $f \in L^{p}(p>1), g \in L^{p^{\prime}}$, then}

(a) $\int_{-\infty}^{\infty} g(t) \cdot B_{\alpha}^{(n)}(f)(t) d t=-\int_{-\infty}^{\infty} f(t) B_{\alpha}^{(n)}(g)(t) d t$,
(b) $\int_{-\infty}^{\infty} g(t) I_{\alpha}^{(n)}(f)(t) d t=-\int_{-\infty}^{\infty} f(t) I_{\alpha}^{(n)}(g)(t) d t$.

Some of the results obtained in the next section depend on the commutative property exibited by each pair of the transforms considered here. In view of this we prove:

THEOREM 10. If $f \in L^{p}(p>1)$, then 
(a) $B_{a}^{(n)}\{X(f)\}=X\left\{B_{a}^{(n)}(f)\right\}$;

(b) $I_{\alpha}^{(n)}\{X(f)\}=X\left\{I_{\alpha}^{(n)}(f)\right\}$, where $X$ represents of the operators $D_{\beta}^{(m)}, S_{\beta}^{(m)}$ or $H$.

Proof. Since $B_{\alpha}^{(n)}(f)-\alpha^{2 n+1} H(f)$ and $I_{\alpha}^{(n)}(f)-\alpha^{2 n+2} H(f)$ define uniformly convergent integrals, the results

$$
X\left\{B_{\alpha}^{(n)}(f)-\alpha^{2 n+1} H(f)\right\}=B_{\alpha}^{(n)}\{X(f)\}-\alpha^{2 n+1} H\{X(f)\}
$$

and

$$
X\left\{I_{\alpha}^{(n)}(f)-\alpha^{2 n+2} H(f)\right\}=I_{\infty}^{(n)}\{X(f)\}-\alpha^{2 n+2} H\{X(f)\}
$$

$\left(X=D_{\beta}^{(m)}\right.$ or $S_{\beta}^{(m)}$ ) follow by obvious inversion of integrals. Theorems 10(a) and (b) for $X=D_{\beta}^{(m)}$ or $S_{\beta}^{(m)}$ then follow by applying Theorem 4 . Next we proceed as in the proof of Theorem 4 to show that

$$
H\left\{B_{a}^{(n)}(f)\right\}+\alpha^{2 n+1} f=H\left\{B_{a}^{(n)}(f)-\alpha^{2 n+1} H(f)\right\}=B_{a}^{(n)}\{H(f)\}+\alpha^{2 n+1} f
$$

and

$$
H\left\{I_{\alpha}^{(n)}(f)\right\}+\alpha^{2 n+2} f=H\left\{I_{\alpha}^{(n)}(f)-\alpha^{2 n+2} H(f)\right\}=I_{\alpha}^{(n)}\{H(f)\}+\alpha^{2 n+2} f .
$$

The case $X=H$ clearly follows.

5. Reduction of $B_{\alpha}^{(n)}(f)$ and $I_{\alpha}^{(n)}(f)$. The results of this section give conditions under which the operators $B_{\infty}^{(n)}, I_{\alpha}^{(n)}$ and $B_{a}^{2(n)}, I_{\alpha}^{3(n)}$ reduce to the Hilbert transform and the identity

THEOREM 11. If $f \in L^{p}(p>1)$ and if $n \geqq 0$, then (a) $B_{a}^{(n)}(f)=\alpha^{2 n+1} H(f)$ if and only if $D_{\alpha}^{(n)}(f)=0$.

(b) If $I_{a}^{(n)}(f)=0$, then $B_{a}^{2(n)}(f)=-\alpha^{4 n+2} f$.

Proof. Since $D_{\alpha}^{(n)}(f)=0$ implies that $S_{\alpha}^{(n)}(f)=0$ (Corollary 3.1), it follows from Theorem 6 that $B_{a}^{(n)}(f)=\alpha^{2 n+1} H(f)$ whenever $D_{a}^{(n)}(f)=0$. Also, under this condition, we have

$$
B_{a}^{2(n)}(f)=\alpha^{2 n+1} B_{\alpha}^{(n)}\{H(f)\}=-\alpha^{4 n+2} f(\text { by }(8)) .
$$

Now suppose that $B_{\alpha}^{(n)}(f)=\alpha^{2 n+1} H(f)$. Then by Theorem 10, we have

$$
B_{\alpha}^{(n)}\{H(f)\}=H\left\{B_{\alpha}^{(n)}(f)\right\}=-\alpha^{2 n+1} f,
$$

so that Theorem 6 and result (8) yield

$$
\begin{aligned}
-\alpha S_{\alpha}^{(n)}(f)+\frac{d}{d x} D_{\alpha}^{(n)}(f) & =0, \\
\alpha D_{\alpha}^{(n)}(f)+\frac{d}{d x} S_{\alpha}^{(n)}(f) & =0 .
\end{aligned}
$$


These equations clearly lead to

$$
\frac{d^{2}}{d x^{2}} D_{\alpha}^{(n)}(f)+\alpha^{2} D_{\alpha}^{(n)}(f)=0 \text {. }
$$

The only solution of this equation for which $D_{\alpha}^{(n)}(f) \in L^{p}$ is clearly given by

$$
D_{\alpha}^{(n)}(f)=0
$$

Note. It has been proved (see [4]) that for $n=0$, the converse of Theorem 11(b) also holds.

THEOREM 12. If $f \in L^{p}(p>1)$ and if $n \geqq 0$, then

(a) $I_{a}^{(n)}(f)=\alpha^{2 n+2} H(f)$ if and only if $D_{\alpha}^{(n)}(f)=0$.

(b) If $D_{\alpha}^{(n)}(f)=0$, then $I_{\alpha}^{2(n)}(f)=-\alpha^{4 n+4} f$.

Proof. By proceeding as in the proof of Theorem 11 and applying Corollary 3.1 and Theorem 7 , it follows that $I_{\alpha}^{(n)}(f)=\alpha^{2 n+2} H(f)$ if $D_{a}^{(n)}(f)=0$. Also, under this condition, we have

$$
I_{\alpha}^{2(n)}(f)=\alpha^{2 n+2} I_{\alpha}^{(n)}\{H(f)\}=-\alpha^{4 n+4} f(\text { by }(11)) .
$$

Now suppose that $I_{\alpha}^{(n)}(f)=\alpha^{2 n+2} H(f)$. Then by Theorem 10, we have

$$
I_{\alpha}^{(n)}\{H(f)\}=H\left\{I_{\alpha}^{(n)}(f)\right\}=-\alpha^{2 n+2} f .
$$

Hence Theorem 6 and result (11) yield

$$
\begin{aligned}
-\alpha^{2} S_{\alpha}^{(n)}(f)+2 \alpha \frac{d}{d x} D_{\alpha}^{(n)}(f)+\frac{d^{2}}{d x^{2}} S_{\alpha}^{(n)}(f) & =0, \\
\alpha^{2} D_{\alpha}^{(n)}(f)+2 \alpha \frac{d}{d x} S_{\alpha}^{(n)}(f)-\frac{d^{2}}{d x^{2}} D_{\alpha}^{(n)}(f) & =0 .
\end{aligned}
$$

On eliminating $S_{\alpha}^{(n)}(f)$, we obtain

$$
\left(\frac{d^{2}}{d x^{2}}-(1+\sqrt{2})^{2} \alpha^{2}\right)\left(\frac{d^{2}}{d x^{2}}-(1-\sqrt{2})^{2} \alpha^{2}\right) D_{\alpha}^{(n)}(f)=0 \text {. }
$$

The only nonvanishing solution of this equation belonging to $L^{p}$ is of the form

$$
D_{\infty}^{(n)}(f)=A e^{-\beta_{1}|x|}+B e^{-\beta_{2}|x|}
$$

where $\beta_{1}$ and $\beta_{2}$ are positive, and $A$ and $B$ are arbitrary constants. Now it is easily seen, by applying Theorem 1(a), that

$$
D_{\alpha}\left(e^{-\beta|t|}\right)(x)=\frac{\beta}{\pi} \int_{-\alpha}^{\alpha} \frac{e^{-i x t}}{\beta^{2}+t^{2}} d t .
$$


Since we require $D_{\alpha}\left\{D_{\alpha}^{(n)}(f)\right\}=D_{\alpha}^{(n)}(f)$ however, we must have $A=B=0$.

Note. It has been proved by the author that for $n=0$, the converse of Theorem $12(\mathrm{~b})$ holds.

6. The inversion processes. Formulae have been obtained by which $f$ may be expressed in terms of either of the transforms $B_{\alpha}(f)$ or $I_{\alpha}(f)$. In this section, we shall express $f$ in terms of the $n$th order integrals under restricted conditions. In the first case we assume that $f$ satisfies $D_{o}^{(n)}(f)=0$. The other formulae involved express $f$ in terms of the $n$th order integrals and the operators of order 0 . The general case in which $f$ is expressed in terms of the $n$th order integrals only will not be treated here since the procedure involves the solution of complicated equations for expressing $D_{a}^{(n)}(f)$ in terms of $D_{\alpha}(f)$. (see Corollary 6.1).

THEOREM 13. Suppose that $f \in L^{p}(p>1)$, and denote $B_{\alpha}^{(n)}(f)$ by $f_{n}^{*}$. Then

(a) for any integer $n \geqq 0$, we have

$$
f=\alpha^{-2 n-1}\left\{S_{\alpha}\left(f_{n}^{*}\right)-H\left(f_{n}^{*}\right)\right\}+\int_{\rightarrow-\infty}^{x} D_{\alpha}\left(f^{*}\right)(t) d t,
$$

(b) if we also have $D_{\alpha}^{(n)}(f)=0$, then

$$
f=\alpha^{-2 n-1}\left\{S_{\alpha}\left(f_{n}^{*}\right)-H\left(f_{n}^{*}\right)\right\} .
$$

Proof. By putting $m=0$ in Theorem 8(a), we obtain

$$
B_{\alpha}^{(n)}\left\{S_{\alpha}(f)\right\}-B_{\alpha}^{(n)}\{H(f)\}=\alpha^{2 n+1} f-\alpha^{2 n+1} D_{\alpha}(f) .
$$

Hence by Theorem 10,

$$
S_{\alpha}\left(f_{n}^{(*)}\right)-H\left(f_{n}^{*}\right)=\alpha^{2 n+1} f-\alpha^{2 n+1} D_{\alpha}(f) .
$$

To complete the proof of (a), we express $D_{\alpha}(f)$ in terms of $f^{*}$. On putting $m=n=0$ in (6) and using Theorem 10, we have

$$
D_{\alpha}\left(f^{*}\right)=\frac{d}{d x} D_{\alpha}(f) .
$$

Since $D_{\alpha}(f) \in L^{p}, \lim _{x \rightarrow \pm \infty} D_{\alpha}(f)(x)=0$. Hence

$$
D_{\alpha}(f)(x)=\int_{\rightarrow-\infty}^{x} D_{\alpha}\left(f^{*}\right)(t) d t
$$

Now suppose that $D_{\alpha}^{(n)}(f)=0$. Then on putting $m=n$ in Theorem 8(a) and using Theorem 10, we have

$$
S_{\alpha}^{(n)}\left(f_{n}^{*}\right)-H\left(f_{n}^{*}\right)=\alpha^{2 n+1} f-\alpha^{2 n+1} D_{\alpha}^{(n)}(f) .
$$


Hence

$$
f=\alpha^{-2 n-1}\left\{S_{\alpha}^{(n)}\left(f_{n}^{*}\right)-H\left(f_{n}^{*}\right)\right\} .
$$

Note. It should be observed that Theorem 13(b) has been written in its present form for convenience. In fact, we have $S_{\alpha}^{(n)}\left(f_{n}^{*}\right)=$ $B_{a}^{(n)}\left\{S_{a}^{(n)}(f)\right\}=0$ since $D_{a}^{(n)}(f)=0$.

In Remark 1, we observed that if $f \in L^{p} \quad(1<p \leqq 2)$ and if $D_{\alpha}^{(n)}(f)=0$, then $D_{\alpha}^{(l)}(f)=0$ for all $l \geqq 0$. We can now deduce this result for the case $p>2$.

Corollary 13.1. If $f \in L^{p}(p>1)$ and if $D_{\alpha}^{(n)}(f)=0$, then

$$
D_{\alpha}^{(1)}(f)=0 \quad \text { for all integers } 1 \geqq 0 \text {. }
$$

Proof. From Theorems 13(a) and (b) it is clear that $D_{\alpha}^{(n)}(f)=0$ implies that $D_{\alpha}\left(f^{*}\right)=0$. Hence it follows from (12) that $D_{\alpha}(f)=0$. Corollary 13.1 now follows from Corollary 3.1.

THEOREM 14. Suppose that $f \in L^{p}(p>1)$ and let $I_{\alpha}^{(n)}(f)$ be denoted by $\tilde{f}_{n}$. Then

(a) for any integer $n \geqq 0$, we have

$$
f=\alpha^{-(2 n+2)}\left\{S_{\alpha}\left(\tilde{f}_{n}\right)-H\left(\tilde{f}_{n}\right)\right\}+\frac{1}{2} \alpha \int_{\rightarrow-\infty}^{x} \sigma_{\alpha}(\tilde{f})(t) \sin 2 \alpha(t-x) d t
$$

where

$$
\sigma_{\alpha}(\tilde{f})(x)=S_{\alpha}(\tilde{f})(x)-2 \alpha \int_{\rightarrow-\infty}^{x} D_{\alpha}(\tilde{f})(t) d t,
$$

(b) if we also have $D_{\alpha}^{(n)}(f)=0$, then

$$
f=\alpha^{-2 n-2}\left\{S_{\alpha}\left(\widetilde{f}_{n}\right)-H\left(\tilde{f}_{n}\right)\right\} .
$$

Proof. By putting $m=0$ in Theorem 8(b), we have

$$
I_{\alpha}^{(n)}\left\{S_{\alpha}(f)\right\}-I_{\alpha}^{(n)}\{H(f)\}=\alpha^{2 n+2} f-\alpha^{2 n+2} D_{\alpha}(f) .
$$

Hence by Theorem 10,

$$
S_{\alpha}\left(\tilde{f}_{n}\right)-H\left(\tilde{f}_{n}\right)=\alpha^{2 n+2} f-\alpha^{2 n+2} D_{\alpha}(f) .
$$

We shall now express $D_{\alpha}(f)$ in terms of $\tilde{f}$ to complete the proof of (a). On putting $m=n=0$ in (9) and (10) and using Theorem 10, we obtain

$$
D_{\alpha}(\tilde{f})=2 \alpha \frac{d}{d x} D_{\alpha}(f)+\frac{d^{2}}{d x^{2}} S_{\alpha}(f)
$$




$$
S_{\alpha}(\tilde{f})=2 \alpha \frac{d}{d x} S_{\alpha}(f)-\frac{d^{2}}{d x^{2}} D_{\alpha}(f)
$$

Since $D_{\alpha}(f), S_{\alpha}(f),(d / d x) D_{\alpha}(f)$ and $(d / d x) S_{\alpha}(f)$ belong to $L^{p}$, we have

$$
\begin{aligned}
\lim _{x \rightarrow \pm \infty} D_{\alpha}(f)(x) & =\lim _{x \rightarrow \pm \infty} S_{\alpha}(f)(x)=\lim _{x \rightarrow \pm \infty} \frac{d}{d x} S_{\alpha}(f)(x) \\
& =\lim _{x \rightarrow \pm \infty} \frac{d}{d x} D_{\alpha}(f)(x)=0 .
\end{aligned}
$$

On integrating (13), we obtain

$$
\int_{\rightarrow-\infty}^{x} D_{\alpha}(\tilde{f})(t) d t=2 \alpha D_{\alpha}(f)+\frac{d}{d x} S_{\alpha}(f) .
$$

This together with (14) gives

$$
\frac{d^{2}}{d x^{2}} D_{\alpha}(f)+4 \alpha^{2} D_{\alpha}(f)=2 \alpha \int_{\rightarrow-\infty}^{x} D_{\alpha}(\tilde{f})(t) d t-S_{\alpha}(\tilde{f})=-\sigma_{\alpha}(\tilde{f}) .
$$

This equation can be written as

$$
\frac{d}{d x}\left(e^{2 i \alpha x} \frac{d}{d x} D_{\alpha}(f)-2 i \alpha e^{2 i \alpha x} D_{\alpha}(f)\right)=-\sigma_{\alpha}(f)(x) e^{2 i \alpha x} .
$$

Hence

$$
\frac{d}{d x} D_{\alpha}(f)-2 i \alpha D_{\alpha}(f)=-\int_{\rightarrow-\infty}^{x} \sigma_{\alpha}(\tilde{f})(t) e^{2 i \alpha(t-x)} d t
$$

A similar equation is obtained by putting $-i$ for $i$. It is then obvious that

$$
2 \alpha D_{\alpha}(f)=\int_{\rightarrow-\infty}^{x} \sigma_{\alpha}(\widetilde{f})(t) \sin 2 \alpha(t-x) d t .
$$

Now suppose that $D_{\alpha}^{(n)}(f)=0$. Then by putting $m=n$ in Theorem $8(\mathrm{~b})$ and using Theorem 10, we have

$$
S_{\alpha}^{(n)}\left(\tilde{f}_{n}\right)-H\left(\tilde{f}_{n}\right)=-\alpha^{2 n+2}\left(f-D_{\alpha}^{(n)}(f)\right),
$$

and the result (b) of the theorem follows.

7. Note on the continuous analogue of the operators. In this section, we define continuous analogues of the operators studied above which preserve the main properties of the integral transforms. This is done by defining kernels similar to those given in Section 1, and which reduce to these when the parameter involved is an integer. Hence for all $\nu \geqq 0$, we define $s_{\nu}(x)$ and $c_{\nu}(x)$ by 


$$
s_{\nu}(x)=\frac{1}{2 \pi} \int_{-\alpha}^{\alpha}(\alpha-|t|)^{2 \nu} e^{i x t} d t
$$

and

$$
c_{\nu}(x)=\frac{-i}{2 \pi} \int_{-\alpha}^{\alpha}(\alpha-|t|)^{2 \nu} \frac{|t|}{t} e^{i x t} d t
$$

It is clear, by Lemma 2 that these kernels reduce to those already given when $\nu$ is an integer. By applying known results involving the Fourier transform of a Hilbert transform, it also follows that results similar to those given in Lemma 1 hold for $s_{\nu}(x)$ and $c_{\nu}(x)$. It is then obvious by procedure similar to that employed above, that if the operators are defined as in Section 1 with $\nu$ in place of $n$, then the results proved with respect to the discrete parameter also hold for the continuous case. In particular, from Theorem 3(a), it follows that for all $\nu \geqq 0$ and $\mu \geqq 0$, we have

$$
D_{\alpha}^{(\nu)}\left\{D_{\alpha}^{(\mu)}(f)\right\}=D_{\alpha}^{(\nu+\mu)}(f),
$$

so that $\left\{D_{\alpha}^{(\nu)}\right\} 0<\nu \leqq \infty$ forms a family of 'strongly continuous semigroup of operators'. Note that in this case we do not have $D_{a}^{(0)}=I$.

\section{REFERENCES}

1. R. P. Boas, Some theorems on Fourier transforms and conjugate trigonometric integrals, Trans. Amer. Math. Soc. 40 (1936), 287-308.

2. R. R. Goldberg, An integral transform related to the Hilbert transform, London Math. Soc. 35 (1960), 200-204.

3. P. Heywood, On a transform discussed by Goldberg, J. London Math. Soc. 38 (1963), 162-168.

4. H. Kober, An operator related to Hilbert transforms and to Dirichlet's integrals,

J. London Math. Soc. 39 (1964), 649-656.

5. E. C. Titchmarsh, The theory of Fourier integrals, Oxford, 1937.

Received April 15, 1965.

The University of Sussex

BRIGHTON, ENGLAND 


\section{PACIFIC JOURNAL OF MATHEMATICS}

\section{EDITORS}

H. SAMELSON

Stanford University

Stanford, California

R. M. BLUMENTHAL

University of Washington

Seattle, Washington 98105

\section{*J. DugundJI}

University of Southern California Los Angeles, California 90007

RICHARD ARENS

University of California

Los Angeles, California 90024

\section{ASSOCIATE EDITORS}

E. F. BECKENBACH
F. WOLF

K. YosIDA

\section{SUPPORTING INSTITUTIONS}

\author{
UNIVERSITY OF BRITISH COLUMBIA \\ CALIFORNIA INSTITUTE OF TECHNOLOGY \\ UNIVERSITY OF CALIFORNIA \\ MONTANA STATE UNIVERSITY \\ UNIVERSITY OF NEVADA \\ NEW MEXICO STATE UNIVERSITY \\ OREGON STATE UNIVERSITY \\ UNIVERSITY OF OREGON \\ OSAKA UNIVERSITY \\ UNIVERSITY OF SOUTHERN CALIFORNIA
}

\author{
STANFORD UNIVERSITY \\ UNIVERSITY OF TOKYO \\ UNIVERSITY OF UTAH \\ WASHINGTON STATE UNIVERSITY \\ UNIVERSITY OF WASHINGTON \\ AMERICAN MATHEMATICAL SOCIETY \\ CHEVRON RESEARCH CORPORATION \\ TRW SYSTEMS \\ NAVAL ORDNANCE TEST STATION
}

Mathematical papers intended for publication in the Pacific Journal of Mathematics should be typewritten (double spaced). The first paragraph or two must be capable of being used separately as a synopsis of the entire paper. It should not contain references to the bibliography. Manuscripts may be sent to any one of the four editors. All other communications to the editors should be addressed to the managing editor, Richard Arens at the University of California, Los Angeles, California 90024 .

50 reprints per author of each article are furnished free of charge; additional copies may be obtained at cost in multiples of 50 .

The Pacific Journal of Mathematics is published monthly. Effective with Volume 16 the price per volume (3 numbers) is $\$ 8.00$; single issues, $\$ 3.00$. Special price for current issues to individual faculty members of supporting institutions and to individual members of the American Mathematical Society: $\$ 4.00$ per volume; single issues $\$ 1.50$. Back numbers are available.

Subscriptions, orders for back numbers, and changes of address should be sent to Pacific Journal of Mathematics, 103 Highland Boulevard, Berkeley 8, California.

Printed at Kokusai Bunken Insatsusha (International Academic Printing Co., Ltd.), No. 6, 2-chome, Fujimi-cho, Chiyoda-ku, Tokyo, Japan.

PUBLISHED BY PACIFIC JOURNAL OF MATHEMATICS, A NON-PROFIT CORPORATION

The Supporting Institutions listed above contribute to the cost of publication of this Journal, but they are not owners or publishers and have no responsibility for its content or policies.

* Paul A. White, Acting Editor until J. Dugundji returns. 


\section{Pacific Journal of Mathematics}

\section{Vol. 18, No. 2 \\ April, 1966}

Alexander V. Arhangelskii, On closed mappings, bicompact spaces, and a problem of P. Aleksandrov .............................. 201

A. K. Austin, A note on loops . . . . . . . . . . . . . . . . . . . . . . . . . . . . 209

Lawrence Peter Belluce and William A. Kirk, Fixed-point theorems for families of contraction mappings...................... 213

Luther Elic Claborn, Every abelian group is a class group ............ 219

Luther Elic Claborn, A note on the class group .................. 223

Robert Stephen De Zur, Point-determining homomorphisms on multiplicative semi-groups of continuous functions............. 227

Raymond William Freese, A convexity property ................ 237

Frederick Paul Greenleaf, Characterization of group algebras in terms of their translation operators ......................... 243

Andrzej Hulanicki, On the spectral radius of hermitian elements in group algebras....................................... 277

Michael Bahir Maschler and Bezalel Peleg, A characterization, existence proof and dimension bounds for the kernel of a game ............ 289

Yiannis (John) Nicolas Moschovakis, Many-one degrees of the predicates

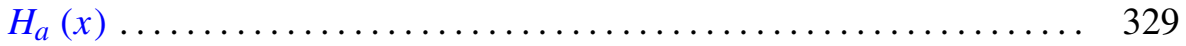

G. O. Okikiolu, $n$th order integral operators associated with Hilbert transforms.

C. E. Rickart, Analytic phenomena in general function algebras ... 361

K. N. Srivastava, On an entire function of an entire function defined by Dirichlet series

Paul Elvis Waltman, Oscillation criteria for third order nonlinear differential equations .......................... 